Perspectiva Geográfica

ISSN 0123-3769 (Impreso) - 2500-8684 (En línea)

Vol. 21 N. ${ }^{\circ} 2$ de 2016

Julio - Diciembre

pp. 299-320

\title{
Proceso migratorio de la comunidad del cabildo Nasa Uka Wesx Thäj: factores, trayectorias espaciales y temporalidades ${ }^{1}$
}

\author{
Migration Process of Nasa Uka Wesx Thäj Community: Factors, \\ Spatial Trajectories and Temporalities
}

\section{Pamela Núñez Basante ${ }^{2}$ \\ Liliana Tálaga Castro ${ }^{3}$}

Para citar este artículo utilice el nombre completo así:

Núñez, P. y Tálaga, L. (2016). Proceso migratorio de la comunidad del cabildo Nasa Uka Wesx Thäj: factores, trayectorias espaciales y temporalidades. Perspectiva Geográfica, 21(2), 299320. doi: $10.19053 / 01233769.5855$

\section{Resumen}

La población indígena de Colombia ha adoptado nuevas formas de movilidad en las últimas décadas a causa del desplazamiento forzado y la búsqueda de oportunidades tanto laborales como educativas. Este artículo fue desarrollado en el ámbito de la comunidad indígena Nasa perteneciente al cabildo Uka Wesx Tháj, oriundos del departamento del Cauca y ubicado actualmente en la zona de ladera suroccidental de la ciudad de Santiago de Cali, Valle del Cauca. El enfoque metodológico utilizado se basa en técnicas cualitativas, a través de

1 Wët Wët Fizenxi Kiwe o el buen vivir en el territorio: Proceso de migración y apropiación socioespacial de la comunidad indígena del cabildo Nasa Uka Wesx Tháj en la ladera suroccidental de la ciudad Santiago De Cali, Trabajo de grado presentado para optar por el título de profesional en Geografía en la Universidad del Valle.

2 Geógrafa, Universidad del Valle. Estudiante de maestría en Ciencias Geográficas en la Vrije Universitet Brussels. pamela. nunez.b@gmail.com

3 Geógrafa, Universidad del Valle. lilinita-30@hotmail.com 
entrevistas a profundidad e investigaciones de acción participativa. El objetivo fundamental de este estudio consistió en analizar el proceso migratorio por parte de la comunidad indígena en el camino hacia la legalización del cabildo, como proceso de reivindicación y fortalecimiento cultural desde el año 2010 hasta la actualidad. El proceso migratorio se dio a través de redes familiares influenciadas por la búsqueda de oportunidades tanto laborales como educativas además del desplazamiento forzado provocado por las olas de violencia que se dieron lugar en el Departamento del Cauca.

Palabras clave: Cabildo Nasa Uka Wesx Thaj, Cali, identidad, indígenas Nasa, migración, redes familiares.

\begin{abstract}
The indigenous population in Colombia has adopted new forms of mobility in recent decades, given the context of displacement caused by armed conflict, job opportunities and education opportunities. This paper was developed in the context of the Nasa native community belonging to the local government council called Uka Wesx Thaj, a group of natives from the Cauca region, currently located at the southwestern side of the city of Santiago de Cali. The methodological approach was based on qualitative techniques through indepth interviews and research on participatory action. The subject of this paper is to analyze the migratory process by the above referenced native community towards a local government council legalization as a process of revindication and cultural strengthening from 2010 until today. This study found that the migratory process was, through family networks, influenced by the search of education and job opportunities besides the forced displacement caused by waves of violence experienced in the Cauca region.
\end{abstract}

Keywords: Nasa Uka Wesx Thaj, Cali, identity, natives, migration, family networks. 


\section{Introducción}

El término Wët Wët Fizenxi Kiwe significa en castellano "Vivir y estar bien, o vivir en alegría en el territorio", este término recoge las orientaciones de un mundo de vida armónico entre todos los seres de la naturaleza. En el mundo andino se conoce como Sumak Kawsay y en castellano se ha traducido como "buen vivir". Por el contrario, aquello que se opone al Wët wët fizenxi kiwe, es el capitalismo, el mal vivir, que degrada el corazón humano y rompe las relaciones en la comunidad (ACIN, 2004).

El presente artículo se realiza en el marco de la elaboración del Plan de Vida del Cabildo indígena Nasa Uka Wesx Thăj, cuya misión es orientar desde la sabiduría Nasa sus prioridades de desarrollo propio para el buen vivir. Esta investigación se integra a dicho plan al evidenciar las necesidades de la comunidad, y al ofrecer mayor coherencia al proceso territorial ante las autoridades del Estado colombiano, todo esto, a través de la actualización del censo poblacional de la comunidad, la geo-referenciación del asentamiento y la formulación de pautas para el manejo pertinente de su situación actual.
La comunidad del Cabildo indígena Nasa Uka Wesx Thăj se ubica en la ladera de los barrios Alto Nápoles y Cuarteles Nápoles de la ciudad de Santiago de Cali, son indígenas oriundos del departamento del Cauca que han migrado de sus territorios de origen por el conflicto armado que se vive al interior de estos, y por la falta de oportunidades tanto laborales como educativas. Este proceso migratorio se llevó a cabo a partir del 22 de septiembre del 2009, cuando indígenas de los resguardos de Pioya, Pueblo Nuevo, San Lorenzo, Quichaya y Pitayo, de los municipios de Caldono y Silvia (Departamento del Cauca), se asentaron en la ladera suroccidental de Cali de acuerdo a su visión cósmica y en uso de sus atribuciones legales y legitimas conferidas por la comunidad. Estas atribuciones son: la Ley de origen, la Ley natural, el derecho mayor, la Ley 89 de 1890, la Constitución Política de Colombia del año 1991 y el convenio 169 de la OIT. Sumado a ello, las comunidades se vieron orientadas por la búsqueda de la vida y su pertinencia a un pueblo originario, y por superar algunas necesidades básicas como la vivienda propia, la educación y las oportunidades laborales. 
El estudio tiene como objetivo general analizar el proceso migratorio y de apropiación socio-espacial de la comunidad indígena del Cabildo Nasa Uka Wesx Thaj en la ladera suroccidental del municipio de Santiago de Cali. El estudio se desarrolló en el período de junio del año 2013 a mayo del año 2014. En la metodología se manejaron técnicas cualitativas de investigación tales como entrevistas a profundidad, observación, recorridos en Tierradentro, Cauca (lugar de origen y territorio ancestral de la comunidad Nasa), recopilación de información secundaria, trabajo de campo (geo-referenciación) y elaboración cartográfica. El análisis se realizó a partir de la herramienta de análisis de contenido.

El artículo se ha estructurado en tres partes, en el primer apartado se presenta el planteamiento del problema; el segundo apartado conforma la metodología, donde se describe el enfoque metodológico, la contextualización de la investigación, la herramienta análisis de contenido utilizada para el análisis de la información; y por último, en el tercer apartado se presenta el análisis de la información y los resultados obtenidos en la investigación, esta posee una estructu- ra conformada por datos (relatos, entrevistas), seguida del análisis (análisis de contenido) y los resultados.

\section{Descripción y planteamiento del problema}

La migración de la población indígena hacia las ciudades es un nuevo fenómeno que adquiere relevancia y se acrecienta con el impacto del conflicto armado, que continuamente alimenta el desplazamiento forzado de dicha población. Según el censo general del año 2005, la población indígena concentrada en los cascos urbanos del país alcanza un total de 298.219 personas, de las cuales 198.424 están concentradas en las ciudades capitales como es el caso de Bogotá, Medellín, Cali, Sincelejo, Riohacha, Valledupar, Inírida, Mocoa, Popayán y Pereira (Echeverry, 2012). En el Departamento del Cauca el conflicto interno y el desplazamiento indígena se caracteriza por la presencia histórica de diversos actores armados, como las Farc, el ELN, el EPL, el M-19, el movimiento Quintin Lame, entre otros (Ávila, 2009). Lo anterior presenta una situación compleja debido a las confrontaciones sociales consecuencia de los altos niveles de pobreza, ade- 
más de las disputas por la tierra entre colonos, campesinos, indígenas y afro colombianos (Ávila, 2009). La magnitud del problema del desplazamiento indígena, que se acrecienta cada día, debe entenderse no solo por las condiciones históricas, sociales, económicas y políticas por las que ha atravesado el departamento en general y sus regiones en particular, sino también por todo el engranaje estructural del país que lo ha motivado e incentivado. En este amplio sentido se debe hacer referencia a las etnias $\mathrm{y}$ a los territorios que habitan para, de esta forma, comprender las dinámicas y las causas particulares del desplazamiento, como también sus consecuencias e impactos en la región o en municipios de expulsión y recepción. Siguiendo a Lapenda (2010), "[a] medida que la migración se consolida, se fortalecen mecanismos de asociación o rechazo, y el espacio habitado puede ser reflejo de tensiones institucionales y culturales" (p. 3). En ese sentido, y siguiendo a Bernal (2012), al trasladarse a los contextos urbanos el pueblo Nasa desea recrear su identidad étnica, su memoria colectiva y su territorialidad. Para el estudio se toma como centro de investigación el territorio de la comunidad perteneciente al
Cabildo Nasa Uka Wesx Thaj, que comprende la zona de ladera de los barrios Alto Nápoles y Cuarteles Nápoles pertenecientes a la comuna 18 de Santiago de Cali, Valle del Cauca. Como ya se anotó, esta zona fue urbanizada principalmente de manera informal, y su población vive en condiciones socioeconómicas por debajo de la ciudad formal, lo cual se ve reflejado en la predominancia del estrato uno.

Derivado de la situación expuesta, es necesario orientar la investigación a través del siguiente cuestionamiento: ¿Cómo ha sido el proceso migratorio y de apropiación socio-espacial de la comunidad indígena Nasa Uka Wesx Thaj, asentada en la ladera suroccidental entre los barrios Alto Nápoles y Cuarteles Nápoles, de la comuna 18 en la ciudad de Cali, desde el año 2009 hasta el presente? 


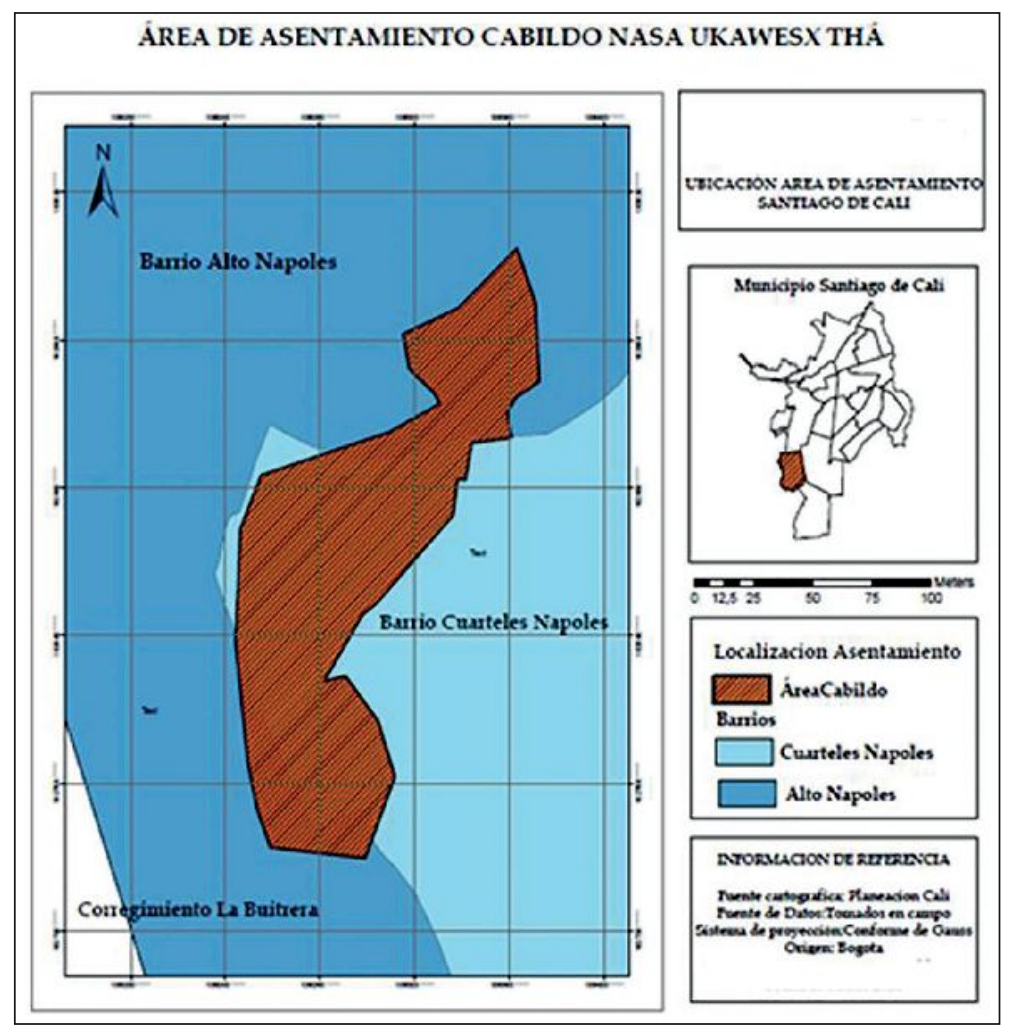

Figura 1. Área de asentamiento Cabildo Nasa Uka Wesx Thaj Fuente: Elaboración propia a partir de Georreferenciación.

\section{Metodología}

\subsection{La investigación acción parti- cipativa (IAP)}

La investigación acción participativa (IAP) fue la base conceptual de la presente investigación; a través de esta fue posible acercarnos a las problemáticas reales de la comunidad.

El desarrollo de este artículo tuvo como finalidad acercarse a la comu- nidad Nasa a través de la presencia en escenarios políticos del cabildo, con el fin de reconocer diversos puntos de vista y abordar realidades y experiencias producto de las vivencias de dicha comunidad. Este acercamiento contó con la colaboración específica de dos núcleos de la población: por un lado la comunidad Nasa que se encuentra asentada y concentra sus acciones en los barrios Alto Nápoles y Cuarteles Nápoles, ubicados en la ladera suroccidental de la ciudad de Cali desde 
hace más de cuatro años; por otro lado, el contacto directo con indígenas Nasa en dos rituales sagrados del Saakhelu (fiesta mayor del pueblo Nasa donde se le rinde tributo a la madre tierra), celebrados en los resguardos indígenas de Tóez Cauca y Santa Rosa ${ }^{4}$ en los meses de agosto y septiembre del año 2013, para tener un acercamiento a su cosmovisión y a la concepción de territorio y naturaleza. El acompañamiento a la comunidad de estudio se realizó durante un periodo de aproximadamente ocho meses.

\subsection{Análisis de contenido (AC)}

El análisis de contenido (AC) establece un vínculo entre tres niveles del lenguaje: el nivel de superficie, el nivel analítico y el nivel interpretativo, tal y como se explica en la Tabla 1.

Tabla 1. Niveles de análisis de contenido

\begin{tabular}{|l|l|}
\hline \multicolumn{1}{|c|}{ NIVEL } & CARACTERÍSTICAS \\
\hline Superficie & $\begin{array}{l}\text { Descripción de la } \\
\text { información }\end{array}$ \\
\hline Analítico & $\begin{array}{l}\text { Clasificación, } \\
\text { ordenamiento de la } \\
\text { información }\end{array}$ \\
\hline Interpretativo & $\begin{array}{l}\text { Comprensión y } \\
\text { constitución de sentido }\end{array}$ \\
\hline
\end{tabular}

Fuente: Tomado de Ruiz (2001, pp. 45-60).

4 Territorio ancestral del pueblo Nasa ubicado en Tierradentro departamento del Cauca.
En el nivel de Superficie se esboza el eje temático (Proceso migratorio) que guio la recolección de la información para este artículo. Igualmente, se establece el contexto relacional-cultural (descripción de rasgos personales y culturales) de los entrevistados.

Por otra parte, en el nivel Analítico se organizan los testimonios de acuerdo a codificadores temáticos. Estos testimonios codificados se relacionan en un análisis semántico intertextual, lo que genera la construcción de categorías de cada eje temático.

\subsection{Técnicas utilizadas}

a. La entrevista. El concepto de "entrevista", según Taylor $\mathrm{y}$ Bogdan (1986), se define a partir de una situación cara a cara donde, en una conversación íntima de intercambio reciproco, el informante se convierte en una extensión de nuestros sentidos y asume la identidad de un miembro de su grupo social.

b. Con la finalidad de situar la entrevista en "profundidad", se sigue que: el entrevistador es quien sugiere el campo a explorar, conservando una cierta liber- 
tad en la forma de llevar a cabo la entrevista así como la forma de responder del entrevistado. A través de la entrevista a profundidad se obtuvo el testimonio de aquellos y aquellas que han promovido y gestionado el reconocimiento político y jurídico del Cabildo rural Alto Buena Vista (Nasa Uka Wesx Tháj), el cual fue reconocido por los Mayores de Tierradentro el 1 de enero del año 2010.

c. En total se realizaron seis entrevistas a profundidad y dos entrevistas a personas externas del cabildo (un médico tradicional "The Wala" y un ex gobernador del cabildo Nasa Urbano de Cali), estas entrevistas se realizaron en castellano.

d. Observación. Esta herramienta se utilizó para la aplicación de los procedimientos sobre la explicación teórica que contribuyeron a reforzar las formas de transmisión del conocimiento, propias de la comunidad indígena. Por medio de la observación participante se detectaron situaciones donde se expresan los universos culturales y sociales de la comunidad, además de su situación como indígenas en la ciudad. e. Actualización Censo. Se realizó en el marco de la construcción del plan de vida del Cabildo, con el objetivo de actualizar la información en salud, educación, y número de personas que conforman el Cabildo, para su posterior legalización ante el Ministerio del Interior.

f. Eje temático para la recolección de la información. Para la recolección de la información, su posterior análisis y la construcción e interpretación del proceso de migración de la comunidad indígena Nasa, se planteó un eje temático que se abarca los resultados mediante las entrevistas en profundidad.

\subsection{Proceso migratorio: motiva- ción, desplazamiento e integra- ción}

Según Eisenstad (1954), se considera al proceso migratorio como un acto social compuesto de tres fases: (a) motivación para migrar: comprende las disposiciones o necesidades que pueden haber inducido a la población indígena a abandonar sus lugares de origen para dirigirse a la ciudad de Cali; (b) estructura del proceso migratorio: considera las características de 
la población migrante, sus lugares de partida, las modalidades, rutas y etapas migratorias; (c) integración de los migrantes a la estructura organizacional urbana a través de indicadores como forma de residencia y de trabajo.

Sobre el primer aspecto de la migración, sabíamos que no era un fenómeno reciente ya que según los entrevistados es un proceso gradual pero permanente. Cada uno de los ciclos económicos por los cuales atravesó el Departamento del Cauca, produjo fuertes desplazamientos de indígenas a consecuencia de la violenta irrupción de guerrillas, ejército y demás grupos armados en sus territorios. La migración es un fenómeno recurrente, un hecho históricamente relacionado con el contacto entre indígenas, mestizos y "blancos", indiscernible de la violenta situación de dominación a la que están siendo sometidos.

Aunque las motivaciones para migrar que fueron relatadas no diferirían de muchas otras corrientes de migración (motivos económicos, sociales o políticos), sí suponen ciertas representaciones comunes íntimamente asociadas a la situación de contacto. Si la necesidad de emigrar es en varias ocasiones un producto de las escasas opciones que ofrece el sistema de relaciones que predomina entre indígenas, mestizos y blancos en el departamento, desde la perspectiva de los entrevistados, la migración es vista más como una alternativa a la actual situación de dominación étnica, marcada por condiciones de existencia vistas como inherente al contacto, que como un hecho atractivo de la ciudad.

De acuerdo con el censo y el mapa realizados durante la investigación, la mayoría de los indígenas provienen de los resguardos de Caldono, Pioya y Pueblonuevo, fuertemente vinculados por una extensa red social urbana. Estos se sostienen a través de los lazos familiares mantenidos por los migrantes, mostrando la existencia de una red extensa con gran conectividad, lo que confirma la persistencia de los vínculos establecidos en los resguardos de origen.

El análisis del proceso migratorio permitió abordar las características de la población que se radica en la ciudad de Cali, así como las formas de traslado prevaleciente entre diferentes generaciones de migrantes. Los datos obtenidos por medio de las entrevistas y sus historias 
migratorias y laborales, revelaron que los antiguos migrantes se trasladaron de manera independiente y por etapas, mientras que los migrantes más recientes se desplazaron directamente al asentamiento.

\section{Análisis de la información}

En este apartado se presenta el análisis de la información y los resultados obtenidos en la investigación. Los subtítulos corresponden al desarrollo de cada objetivo específico planteado. Este posee la siguiente estructura: datos (relatos y entrevistas), seguidos del análisis (análisis de contenido) y los resultados parciales de cada objetivo.

\subsection{Proceso migratorio de la co- munidad del Cabildo Nasa Uka Wesx Thaj: Factores, trayecto- rias espaciales y temporalidades}

El objetivo que guía el presente apartado es conocer el proceso migratorio de la comunidad en estudio, incluyendo factores, trayectorias espaciales y temporalidades; teniendo en cuenta una aproximación histórica de las migraciones en Colombia, y específicamente en el Departamento del Cauca.

\subsection{Eje temático categorial: pro- ceso migratorio}

El proceso migratorio se entiende como el tiempo de recorrido y llegada de indígenas Nasa a la ciudad de Cali. Según declaraciones de los comuneros entrevistados, los indígenas llevan un periodo de cuatros años desde el momento en que comenzaron a migrar a la ciudad de Cali: unos por falta de oportunidades laborales, otros por causa de los conflictos armados que se viven en sus territorios, otros por problemas familiares. La mayoría manifiesta haber llegado a los barrios de Meléndez, Nápoles, el Centro y Siloé. Estas personas generalmente llegan donde familiares, quienes llevan viviendo más tiempo en la ciudad. Otros, llegan a los lugares de trabajo como es el caso de las mujeres indígenas que trabajan en el servicio doméstico, en barrios como el Limonar, Alto Nápoles, los Chorros, Cañaveralejo, San Fernando, Valle de Lili y el Ingenio. Así lo manifiesta Alba Luz Cuetochambo (2013):

Cuando llegué a Cali por primera vez vine a trabajar en una casa de familia a cuidar dos niños, yo ya tenía 15 años, yo trabajaba en el barrio Valle del Lili, luego 
estuve trabajando en el barrio Limonar otro años, después, nosotros salíamos los Domingos al parque de las Banderas y allí me encontraba con otras muchachas, que trabajaban en San Fernando, el Limonar, ellas fue que me comentaron del cabildo alto Buena Vista y así fue como me fui a vivir allá con una tía. (Cuetochambo, 2013)

Las primeras 25 personas que llegaron a este asentamiento residían apenas dos años en la ciudad, algunos en el barrio Meléndez, otros en los barrios Los Chorros, y otros en Alto Nápoles.

\subsubsection{Sub-eje temático: redes} familiares y proceso de asentamiento

Una red familiar dentro de la comunidad es el principal lazo conductor del poblamiento en este lugar. Según testimonio de los entrevistados, y en diálogo con los comuneros del Cabildo Nasa Uka Wesx Tháj manifiestan haber llegado a este lugar por influencia de un familiar.

\subsection{Redes familiares de la comu- nidad}

En cuanto al proceso de ocupación del asentamiento indígena, María Eugenia Osnas (2013) comenta:

Aquí cada día llegan más familias, llega el yerno, la nuera, hay casas en donde viven más de cuatro familias, en una sola casa, después llegan los nietos [...] Primero se viene la mamá, el esposo después los hijos y así van llegando todos. (Osnas, 2013)

El poblamiento en el asentamiento se ha dado por redes familiares, donde los apellidos que más predominan son los Chocue, Chilo, Peña y Osnas. Normalmente un núcleo familiar en este asentamiento está conformado por ocho o nueve personas entre padres, hijos y abuelos. Según Jorge Eliecer Peña (2013):

Toda la vida viví en Pueblo Nuevo de Caldono, yo soy de allá [...] primero llegaron unos familiares, ellos tenían una casa de arriendo en Siloé, nosotros veníamos a visitarlos, un día nos dijeron que por acá estaban cogiendo tierras, entonces yo con mi esposa decidimos venirnos, eso fue un día jueves 22 de diciembre del año 2010. Así fue que nos vinimos a vivir a acá 
[...] La mayoría son de Caldono y Pioya, de resto de Pueblo Nuevo hay 5 o 4 familias y de Quichaya hay 5 o 6 familias. Más que todo de Caldono, Jambalo y hablan Nasayuwe, por eso es que llega mucha gente vienen a visitar familias, más que todo el asentamiento está formado por familias. (Peña, 2013)

En el caso de Dora (2013): "la esposa de Jorge es mi sobrina, entonces por eso yo llegué aquí...Yo vine acá porque tengo familia, y los del cabildo me dieron lotesito..." En el caso de la Gobernadora del Cabildo: "tengo dos hijas, la menor ha vivido siempre conmigo; $y$ la mayor sí vivía en el Cauca, hace cuatro años que vive conmigo. En el momento yo tengo más familia que vive acá, mi papá también vive acá” (Osnas, 2013).

\subsection{Proceso de asentamiento de la comunidad}

Cuando los habitantes indígenas llegaron por primera vez, se ubicaron en dos lugares del asentamiento comprendidos entre la zona de ladera de los barrios Alto Nápoles y Cuarteles Nápoles. Según testimonio de Juan Carlos Chindicue, Coordinador guardia del Cabildo:
[C]uentan los mayores, que los primeros compañeros que llegaron acá que fueron 20 personas que vivían por acá cerca, en ese tiempito ellos pagaban arrendo, llevaban su tiempito viviendo por acá alrededor, si no que ya el problema de ellos era que no le alcanzaban sus recursos, lo que ganaban no les alcanzaba para pagar arrendo, la alimentación, cosas así, les quedaba muy complicado a ellos el tema económico, entonces un compañero indígena le propuso el tema de tomarse este predio de acá arriba, ya fueron analizando hasta que ya esas 20 personas entraron, ya esas 20 personas le fueron comentando a otros compañeros del Cauca que estaban acá, les dijeron vengasen y así se fueron viniendo uno detrás de otro...Los compañeros los primeros que llegaron se repartieron equitativamente las tierras, la idea era que todos cupieran en sus casas que quedaran bien, más de uno miró fue más adelante, sabían que la familia se les iba ir creciendo, ellos tomaron las medidas repartieron bien, analizaron bien los caminos, por donde iban hacer los andenes, repartieron por igualdad. (Chindicue, 2013) 
A lo que agrega el capitán del Cabildo:

Siempre que vamos a conseguir un lote, primero hablamos con los mayores, no puede uno entrar, tenemos que ofrecerle algo a la madre tierra para poder estar tranquilos, ya nos dijeron que salimos bien... yo compre este lote, cuando yo llegué esto ya estaba lleno de gente de aquí para arriba, nosotros cuando llegamos ya casi no habían lotes. Ese día si fue duro, cuando yo llegue el agua llegaba con manguerita hasta la cancha y tocaba llegar con baldes a recoger para hacer la comida y todo eso, eran 70 lotes, cogieron agua, otros vecinos que no tenían agua también, nosotros de buena gente fuimos regalando el agua y después ya no alcanzo para todos, y después toco traer otro tubo. (Peña, 2013)

Añade la Gobernadora del Cabildo:

De haber llegado a este asentamiento 4 años que ya cumplimos, yo llegue 15 días después de haber tomado estos lotes; las primeras 20 personas comuneros de otros resguardos que llegaron a este lugar, llegaron un 22 de septiembre del año 2009, yo llegué fue porque me di cuenta que habían comunidades indígenas que se estaban asentado aquí, entonces yo vine a mirar a ver si era cierto, cuando yo ya llegué los vi a ellos y entonces ya eran conocidos, ya yo me fui quedando con ellos, y empecé a trabajar con ellos. El motivo que los trajo a este lugar fue porque ellos no podían seguir pagando más arrendo. De ahí fue que la gente se dio cuenta y así fueron llegando uno tras otro. (Osnas, 2013)

Como se evidencia en los testimonios, los primeros indígenas que poblaron el asentamiento ya llevaban algún tiempo viviendo en la ciudad como arrendatarios, en barrios cercanos. De acuerdo a las entrevistas, es posible identificar que estos 20 indígenas caucanos son los "pioneros" del asentamiento, quienes se establecieron en el lugar de destino y comenzaron a contribuir en el incremento de los flujos migratorios, especialmente de sus familiares indígenas. e destino y comenzaron a distribuioomicos

\section{Resultados y discusión}

La composición demográfica de los miembros del cabildo Nasa 
Uka Wesx Tháj, de acuerdo a la actualización del censo realizado por las investigadoras en el mes de diciembre del año 2013, demuestra que el cabildo Nasa Uka Wesx cuenta con una población de 106 habitantes: $50 \%$ mujeres y $50 \%$ hombres, de los cuales el 85,84\% son indígenas originarios de distintos resguardos del departamento del Cauca, 12,2\% nacidos en Cali, $0,9 \%$ nacidos en Bogotá y $0,9 \%$ nacidos en Jamundí. El 40,15\% de los miembros del cabildo son menores de edad mientras que el 59,85\% son adultos y adultos mayores.

\section{Lengua materna}

Los indígenas de la etnia Nasa, relacionan la lingüística con la palabra "Nasa" que significa "Gente". Se considera que la recuperación de los valores socioculturales Nasas está muy relacionado con la lengua materna, en la medida que la definición de categorías y pensamiento propio depende de las particularidades lingüísticas. Con el transcurrir del tiempo, el proceso de aculturación ocasionado por la migración a la ciudad ha disminuido el uso cotidiano de la lengua. Dado que la comunidad indígena perteneciente al Cabildo Nasa Uka Wesx Tháj pertenece a la etnia
Nasa, la lengua materna predominante es el Nasa Yuwe, donde el $86,7 \%$ la habla y escribe, mientras que el 13,20\% restante manifiesta que su lengua principal es el castellano. Vale la pena recalcar que todos los miembros del cabildo dominan el castellano.

Como se muestra en la Figura 2, la mayoría de indígenas encuestados son nacidos en resguardos del departamento del Cauca. Alrededor de 13 personas nacidas en la ciudad de Cali corresponden a niños nacidos durante los años de conformación del cabildo. Los anteriores datos pueden ser consecuentes con el hecho de que cada territorio se constituye por sí mismo como un punto geográfico para la confluencia de diversas personas, que quieren tomarlo como asentamiento permanente o transitorio, dependiendo de la actividad que deseen desarrollar. Asimismo, como fue expresado por los líderes del cabildo, sus jurisdicciones son espacios abiertos para todos aquellos que deseen habitarlos, sin distingo de procedencia, raza y credo, esto se debe a que consideran que se es indígena por el vivir en un territorio colectivo, y por tanto ningún lugar debe ser vedado para la ubicación y residencia de cualquier familia o persona. 


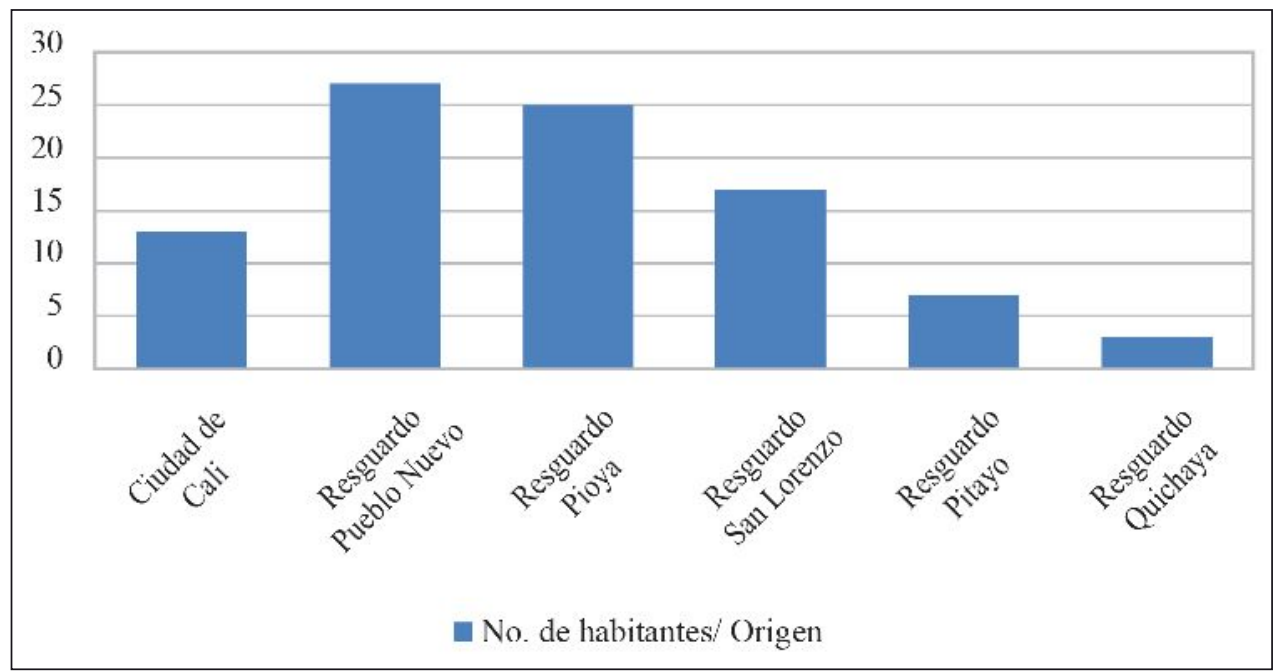

Figura 2. Lugar de nacimiento miembros Cabildo Nasa Uka Wesx Thaj

Fuente: Elaboración propia.

Como se observa en la Figura 3, tamento del Cauca, y por falta de la estructura de localización está oportunidades educativas y de traen función de las redes familiares y recomendaciones del The Wala (quien realiza una ceremonia para que la naturaleza indique el lugar predilecto). Esta afirmación deriva también de las condiciones que señalan las familias para la localización de las viviendas de los hijos y los vecinos: "[c]erca pero no al lado, a la distancia que está ahora, cerca pero no enfrente, cerca de la mía" (Osnas, 2013), como una necesidad de definir su territorialidad. Esto es el resultado de una migración producida por redes familiares, influenciada a su vez por el desplazamiento forzado producto de olas de violencia en el deparbajo. Se considera a las redes familiares como un elemento explicativo del crecimiento de los flujos migratorios, de los comuneros Nasa al asentamiento de estudio, desde hace cuatro años hasta la fecha presente. Las redes familiares cumplen diversas funciones tanto en el resguardo de origen como en el asentamiento, las mismas que facilitarían en muchos casos la puesta en marcha del proyecto migratorio. Este hecho ayuda a explicar el incremento de los flujos migratorios hacia la ciudad de Cali.

Las funciones que desempeñan las redes familiares en el asentamien- 
to adoptan una importancia fundamental, sobre todo en el momento de llegada. La concentración de indígenas Nasa en determinadas zonas de la ciudad de Cali se puede explicar a partir del papel que cumple la red en la selección de dichos lugares, en los cuales se han establecido los primeros emigrantes indígenas Nasa.

Otra de las funciones de gran importancia en el momento de llegada de los emigrantes indígenas Nasa, es la presencia de relaciones de colaboración y ayuda mutua que predominan especialmente en el momento de la inserción a la vida laboral.

Una limitación que viene de la mano de la asistencia que prestan las redes a sus familiares es la reducida gama de posibilidades de salir del círculo creado por ellas. Como se mencionó anteriormente, la comunidad perteneciente al $\mathrm{Ca}$ bildo pretende aislarse lo más posible de la ciudadanía caleña, puesto que influye en la fragmentación de su cosmovisión y prácticas culturales. A esto se suma la situación de irregularidad de los indígenas como migrantes, así, la búsqueda de otras oportunidades en el cam- po laboral estará impedida por la inseguridad que ello genera.

Se tiene entonces que el patrón de asentamiento responde a una organización social basada en la comunidad formada por conjuntos de familias con estrechas relaciones de cooperación. Estas redes tienen la capacidad de multiplicarse porque cada vez se van incorporando nuevos indígenas al cabildo Nasa Uka Wesx Tháj. Estos datos dan a conocer que la emigración no ha cesado, que sigue desarrollándose a pesar de que las condiciones del cabildo son aun inciertas, y que no hay claridad en la implementación y la ejecución de políticas públicas en la ciudad para comunidades indígenas.

En cuanto al tiempo de permanencia en el asentamiento, el siguiente cuadro describe el número de años de permanencia en el asentamiento por cada una de las familias miembros del Cabildo Nasa, de acuerdo al censo realizado por las investigadoras. 


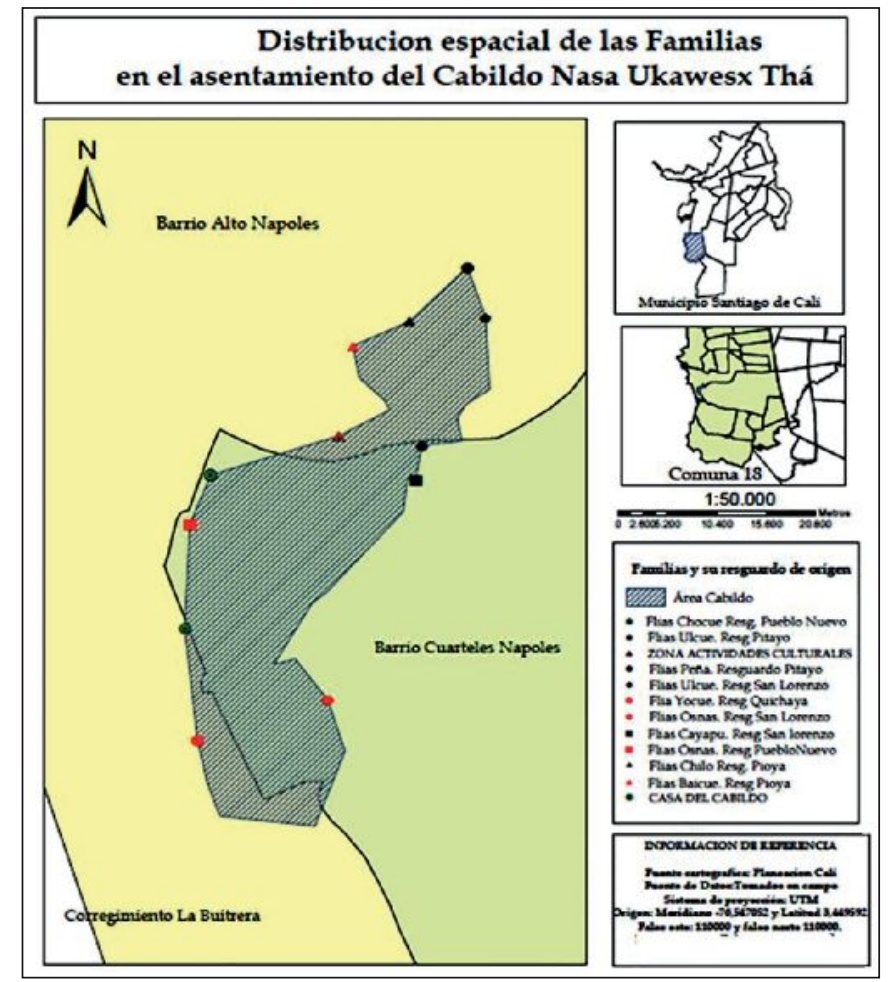

Figura 3. Distribución espacial de las familias en el asentamiento del Cabildo Nasa Uka Wesx Tháj

Fuente: Elaboración propia.

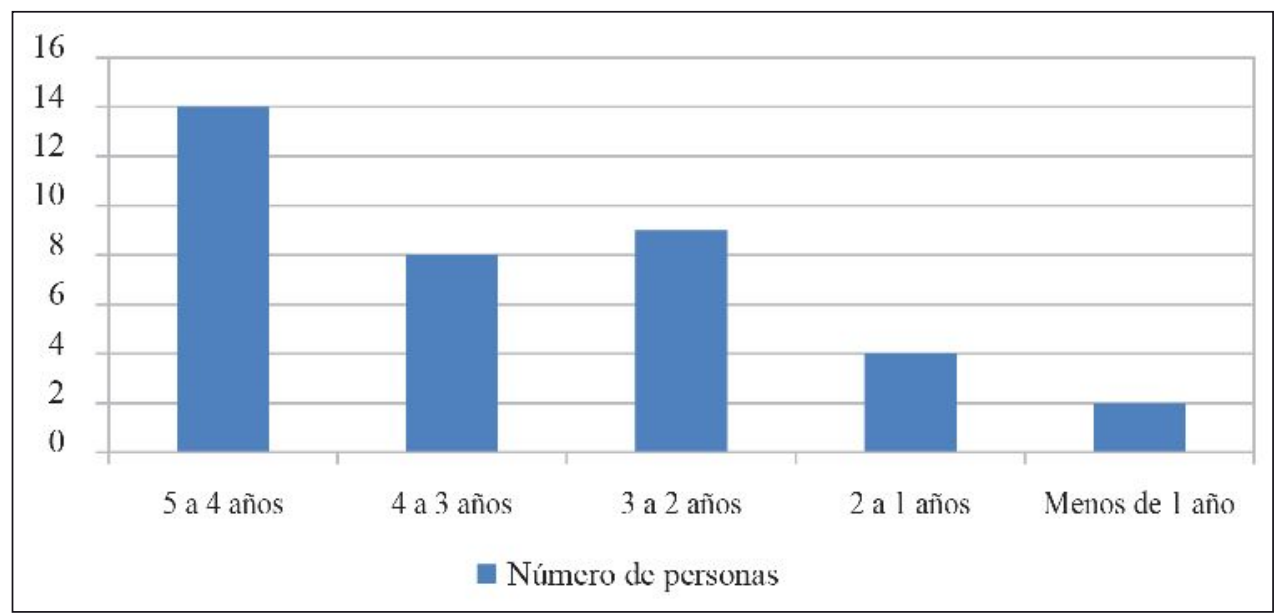

Figura 4. Años de permanencia en el asentamiento

Fuente: Elaboración propia. 
Alrededor del 50\% de las familias encuestadas presentan un tiempo de permanencia en el asentamiento entre cinco y cuatro años. Las proporciones así referidas dan cuenta que el arraigo en el actual lugar de asentamiento es una conquista territorial al lograr mantener cierto grado de estabilidad poblacional entre sus habitantes. No obstante, las situaciones adversas que contravienen las familias, como el hostigamiento frecuente de la Secretaría de Vivienda, atentan sobre la dignidad de las personas.

Uno de los aspectos con los que se podría establecer una aproxi- mación del flujo migratorio poblacional en territorios donde la información secundaria es inexistente, sería en toma de referencia del tiempo de permanencia de las familias al interior de un territorio. Este planteamiento mantendría que cuanto mayor sea la proporción de personas, ubicadas de manera reciente en un territorio, más dominante es el patrón migratorio de la población. Lo anterior muestra una clara tendencia en el incremento del flujo migratorio hacia el asentamiento. En torno a esto, surge una gran preocupación puesto que no se dispone de espacio para nuevas viviendas.

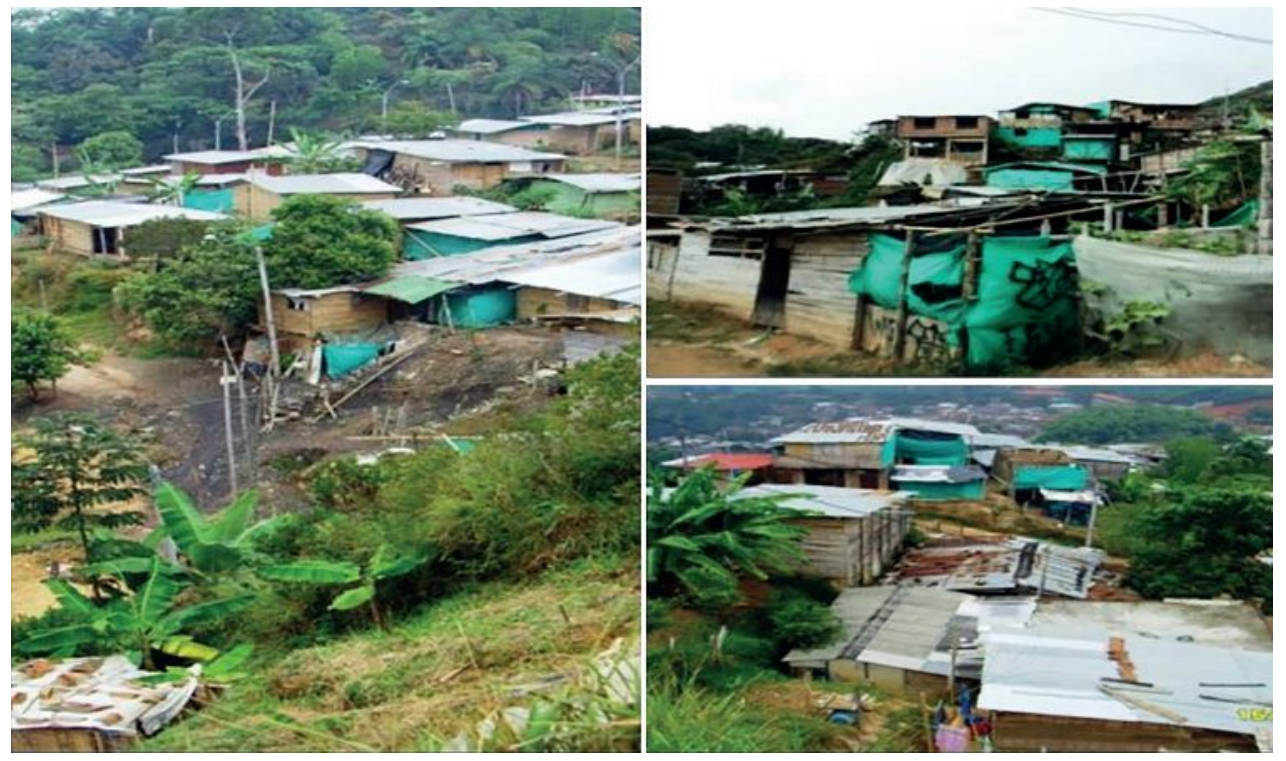

Figura 5. Conglomerado de viviendas en el asentamiento de estudio Fuente: Elaboración propia. 


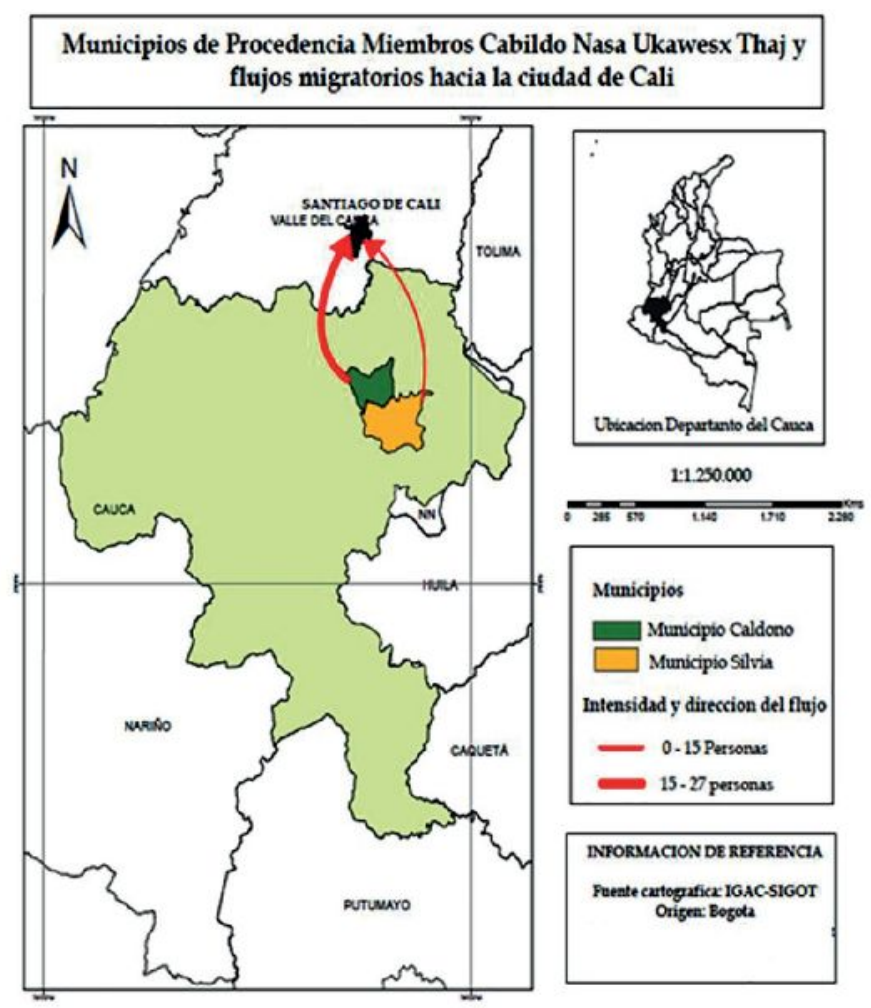

Figura 6. Municipio de procedencia miembros del Cabildo Nasa Uka Wesx Tháj y flujo migratorio hacia la ciudad de Cali

Fuente: Elaboración propia.

En la Figura 6 se espacializa la siendo el municipio de Caldono el información obtenida a través del que presenta el mayor número de censo, ubicando los municipios de migrantes indígenas.

Caldono y Silvia, que contienen dentro de su área municipal los resguardos de Pueblo Nuevo, Quichaya, Pitayo, San Lorenzo y Pioya. Estos se localizan al nororiente del Departamento del Cauca. Como se observa, las direcciones de los flujos migratorios están orientadas hacia la ciudad de Santiago de Cali,

\section{Conclusiones}

La investigación permite afirmar que la migración hacia el asentamiento es producida por redes familiares sometidas por el desplazamiento forzado y por falta de oportunidades educativas y la- 
borales. Las redes familiares son un elemento explicativo del crecimiento de los flujos migratorios de los Nasa al asentamiento de estudio. Asimismo, esta migración a las ciudades no solo implica un desarraigo territorial sino también cultural, que afecta el tejido social $\mathrm{y}$ económico en el contexto de su vida cotidiana. Habitar en la ciudad implica que la vida cotidiana se vea "fragmentada", ya que es necesario vincularse a los diferentes aspectos de la vida urbana, como el empleo, la educación, el servicio de salud, las maneras de esparcimiento, la vestimenta, la tecnología, entre otros. Los miembros del cabildo consumen cada vez más esta serie de bienes adquiridos en la ciudad de Cali, así como nuevas actividades y nuevos intereses -introducidos por el contacto con el mundo citadino-creando nuevos espacios, simbólicos y físicos que también polarizan la vida en la comunidad.

El éxodo masivo de comunidades indígenas hacia los centros urbanos a raíz del desplazamiento forzado, o de la migración económica, agudiza más los problemas sociales que se registran en las ciudades. Los indígenas demandan soluciones a sus necesidades básicas inmediatas, al llegar a la ciu- dad, en la mayoría de los casos los indígenas se convierten en grupos marginales urbanos. Estas recientes movilizaciones poblacionales implican no solo el desarraigo ancestral sino que además comportan nuevas adaptaciones culturales.

Debido a que en la ciudad no hay tierra para producir, es una necesidad vender la fuerza de trabajo, pues este es el único patrimonio para el indígena en la ciudad. Al migrar de sus territorios de origen ubicados en el Departamento del Cauca, los indígenas emprendieron el gigantesco esfuerzo de hacer suyo este asentamiento en la ladera suroccidental de Cali. La única manera de que la recuperación sirviera para afianzar su conocimiento tradicional como etnia era apropiarse de este asentamiento como un territorio propio, y así poder crecer como identidad diferente. En el camino de emprendimiento de esta nueva territorialidad se encontró que existen espacios con gran potencial comunitario donde se concentran actividades como mingas, reuniones, rituales, entre otros. Estos también hacen parte de sus prácticas cotidianas de interacción con otros grupos sociales que habitan en el asentamiento. 


\section{Referencias}

ACIN, Asociación de cabildos Indígenas del Norte del Cauca. (2004). Boletín informativo.

Ávila, A. (2009). Conflicto armado en Cauca: reconfiguración del poder regional de los actores armados. Bogotá D.C.: Corporación Nuevo Arco Iris.

Bernal, M. (2012). Territorialidad Nasa en Bogotá. Apropiación, percepción y sentido de lugar. Cuadernos de Geografia: Revista Colombiana de Geografia, 21(1), 83-98.

Chindicue, J. (15 de enero, 2013). Entrevista con coordinador Guardia del Cabildo indígena Nasa Uka Wesx Tháj. Parte 2. [Entrevista realizada por P. Núñez y L. Tálaga].

Chocue, Dora L. (20 de octubre, 2013). Entrevista con Dora, participante de la comunidad indígena Nasa Uka Wesx Tháj. Parte 1. [Entrevista realizada por P. Núñez y L. Tálaga].

Cuetochambo, A. (17 de noviembre, 2013). Entrevista con Alba Luz Chetochambo, participante de la comunidad indígena Nasa Uka Wesx Tháj. Parte 1. [Entrevista realizada por P. Núñez y L. Tálaga].

Echeverry, H. (2012). Población indígena y las ciudades. Bogotá: Observatorio étnico Cecoin. Recuperado de http://observatorioetnicocecoin.org.co

Eisenstad, S. (1954). The Absorption of Immigrants: A Comparative Study Bases Mainly on the Jewish Community in Palestine and the State of the Israel. London: Routledge and Kegan Paul.

Lapenda, L. (2010). Migrantes peruanos en la ciudad de Buenos Aires. Construcción de lugares y visibilidad. Buenos Aires. www.vocesenelfenix.com. Recuperado de http://www. vocesenelfenix.com/sites/default/files/pdf/14_5.pdf

Osnas, M. (4 de octubre, 2013). Entrevista con gobernadora del Cabildo indígena Nasa Uka Wesx Tháj. Parte 2. [Entrevista realizada por P. Núñez y L. Tálaga]

Peña, E. (26 de octubre, 2013). Entrevista con Capitán del Cabildo indígena Nasa Uka Wesx Tháj. Parte 1. [Entrevista realizada por P. Núñez y L. Tálaga]

Ruiz, A. (2001). Texto, testimonio y metatexto: el análisis de contenido en las investigaciones en educación. Bogotá: Universidad Pedagógica Nacional. 
Recepción: 12 de mayo de 2015

Evaluación: 18 de enero de 2016

Aprobación: 1 de agosto de 2016 